\title{
Rawlsian Pricing of Access to Public Facilities: a Unidimensional Illustration*
}

\author{
Jacques Drèze ${ }^{\dagger} \quad$ Michel Le Breton ${ }^{\ddagger} \quad$ Shlomo Weber ${ }^{\S}$
}

March 2006

\begin{abstract}
We consider a setting where citizens using a public facility (e.g., a hospital, a library or a post-office) face an idiosyncratic private access cost (e.g. transportation) and, in addition, must contribute to the costs of facility. We show that if the population is uniformly spread over the entire real line, if the cost of a facility is independent of location and access costs are linear with respect to distance, then the Rawlsian access pricing (i) equates the total cost borne by every citizen; and (ii) is the unique cost sharing solution that satisfies the "core property" of secession-proofness that amounts to the voluntary participation principle under which no group of citizens should be charged more than the cost incurred if it had acted on its own.
\end{abstract}

Keywords: Optimal jurisdictions, Secession-proofness, Rawlsian allocations, Efficiency.

JEL Classification Numbers: D70, H20, H73.

${ }^{*}$ We thank two anonymous referees and an associate editor for very useful comments and suggestions. This note is a shortened version of Le Breton et al. (2004). An interested reader is invited to consult that longer manuscript that contains additional results.

${ }^{\dagger}$ CORE, Catholic University of Louvain, Belgium.

‡Université de Toulouse I, GREMAQ and IDEI, Toulouse, France.

$\S$ CORE, Catholic University of Louvain, Belgium, and Department of Economics, Southern Methodist University, U.S.A. 


\section{Introduction}

When citizens using a public facility (e.g. a hospital, a library or a post-office) face an idiosyncratic private access cost (e.g. transportation) and must in addition contribute to the fixed costs of the facility, two questions arise:

How does one characterize the optimal number and "locations" of the facilities?

Should the contributions respond, ceteris paribus, to the differences in private cost?

As a simple illustration, ${ }^{1}$ we consider a population that consists of a continuum of citizens uniformly distributed over the real line. Facilities can be located anywhere on the line at a cost independent of location. All citizens face common private access costs proportional to the distance to the chosen facility. For this version of the problem we provide an answer to the two questions raised above. First, we show that there is a uniquely determined optimal "density" of facilities, each serving a connected subset of citizens (called jurisdiction) of the "optimal" size. Then we examine the implications of the core-like pricing principle, called secession-proofness: no group of citizens $S$ should bear a total cost higher than that incurred by $S$ had it acted on its own by creating a separate facility. In other words, the pricing formula should guarantee the voluntary participation of any group of citizens. Our main result asserts, somewhat surprisingly, that the Rawlsian pricing formula implementing full cost equalization is the unique secession-proof allocation. ${ }^{2}$ Under the Rawlsian formula, in each jurisdiction the contribution of a citizen is given by the difference between the average total cost and her private access cost to the facility in the jurisdiction. The Rawlsian policies are often advocated on the basis of justice considerations and sometimes on grounds of secondbest efficiency, e.g., under incomplete markets. Our result adds a stability dimension for the rationale of the Rawlsian principle.

\footnotetext{
${ }^{1}$ In this paper we adopt the spatial interpretation of the horizontal differentiation setting.

${ }^{2}$ Le Breton et al. (2004) show that this result cannot be extended to societies whose population distribution has a finite support, where the Rawlsian principle and secession-proofness are, in general, inconsistent. Le Breton et al. introduce the notion of approximate Rawlsian allocation, under which the proportion of individuals whose cost burden is substantially lighter relative to the Rawlsian allocation is commensurably small, and show that in large finite societies approximate Rawlsian allocations are secession-proof.
} 
As to the related literature, Alesina and Spolaore (1997) examine the existence of secessionproof allocations in the case where the population is uniformly spread over a bounded interval and the unique cost allocation available for each jurisdiction is the equal-share scheme, according to which all citizens in the same jurisdiction make an identical contribution towards the facility cost. Le Breton and Weber (2003) prove the existence and characterize secessionproof cost allocations for a large class of absolutely continuous distributions. Haimanko et al. (2004) establish the existence of secession-proof cost allocations in the general case of an arbitrary probability measure with bounded support.

The paper is organized as follows: in the next section we present the model, state our main result and provide some intuition for it. Section 3 contains the proof of the main result, that relies on several claims and lemmas, proved in the Appendix.

\section{The Model and the Result}

We consider a population of citizens located on the real line $\Re$. The citizens' distribution is given by the Lebesgue measure $\lambda$ over $\Re$. The population is partitioned in several groups (jurisdictions) each providing its members with an access to its own facility. The cost of each facility is independent of the jurisdiction and is given by a positive number $g .{ }^{3}$ A citizen with location at $t$ (for simplicity, labelled $t$ henceforth), who resides in a jurisdiction whose facility location is at $l$, faces a transportation $\operatorname{cost} d(t, l)=|t-l|{ }^{4}$

Let $S$ be a bounded subset of $\Re$ with a positive measure (not necessarily an interval) that defines a jurisdiction. An $S$-cost allocation is a specification of the individual contributions to the fixed cost of the facility, say $x(t)$ for citizen(s) located at $t$. We impose the budgetbalancedness condition, i.e., the total contribution of all members of $S$ is equal to the cost of the facility: $\int_{S} x(t) d t=g$.

\footnotetext{
${ }^{3}$ This assumption can be weakened to include the case where the facility costs are jurisdiction-dependent and are given by $g(S)=g+\alpha \lambda(S)$, where $g$ and $\alpha$ are positive constants.

${ }^{4}$ Again, this can be easily generalized to cover the case where the transportation cost is a continuous and convex function of the distance.
} 
A solution to the allocation problem for the entire population consists of a set of located facilities, jurisdictions (a comprehensive assignment of citizens to facilities), and a cost allocation for every jurisdiction. Since in our set-up every citizen will be assigned to the closest facility, a solution can be described by the pair $(P, x)$, where $P$ is a partition of $\Re$, and $x$ determines a cost allocation for every jurisdiction in $P$. Our goal is to study solutions endowed with a secession-proofness property, ${ }^{5}$ under which the formation of jurisdictions and the choice of cost allocations within each of them rules out the emergence of a potentially seceding group (prone to secession), that can benefit all its members. To proceed with the formal definition, denote by $D(S)$ the minimal aggregate transportation cost within $S$ : $D(S)=\inf _{l \in \Re} \int_{S} d(t, l) d t$. It is easy to see that the minimum is attained when $l=m(S)$, where $m(S)$ is a median of the set $S$. This observation guarantees that every jurisdiction will locate its facility at a median. Therefore, we define:

Definition: Given a solution $(P, x)$, the group $S$ is prone to secession if

$$
\int_{S}(d(t, m(S(t)))+x(t)) d t>D(S)+g
$$

where $S(t)$ is a jurisdiction in $P$ that contains $t$.

If no group is prone to secession, then the pair $(P, x)$ is called secession-proof; if there is no ambiguity we drop the first argument of the pair and simply refer to a secession-proof cost allocation.

The crucial role in our discussion will be played by the Rawlsian $S$-cost allocation $x_{S}^{R}$ which displays complete equalization by making the sum of transportation costs and contribution towards the cost of the facility identical for all members of $S$. Obviously, this allocation minimizes the highest individual cost over $S$. Since the sum of aggregate transportation costs and fixed facility cost faced by $S$ is $D(S)+g$, the contribution of an individual

\footnotetext{
${ }^{5}$ The concept of secession-proofness introduced here is closely related to the notion of the core of a game with coalition structure (Aumann and Drèze (1974)), whose set of players is $\Re$, and the set of feasible outcomes of a coalition $S$ is given by the set of all $S$-cost allocations. Since we do not use the game-theoretical formalism here, we formulate our results without referring to it.
} 
$t \in S$ is:

$$
x_{R}^{S}(t)=\frac{D(S)+g}{\lambda(S)}-d(t, m(S)) \text { a.e. on } S,
$$

where $\lambda(S)$ is the measure of $S$. A cost allocation $x$ attached to the partition $\mathrm{P}$ is called Rawlsian and is denoted $x_{R}^{P}$ when the cost allocation is Rawlsian for every jurisdiction in $P$.

We are now in position to state our main result. Let $s^{*} \equiv 2 \sqrt{g}$ and $P^{*}$ be a partition of $\Re$ into equal intervals of size $s^{*}$. We show that the Rawlsian allocation associated with the partition $P^{*}$, denoted simply by $x_{R}^{*}$, is the $u n i q u e^{6}$ secession-proof allocation.

Theorem: A pair $(P, x)$, where $P$ is a partition of $\Re$ and $x$ is a $P$-cost allocation, is secessionproof if and only if $P=P^{*}$ and $x=x_{R}^{*}$.

In our proof we utilize the concept of an efficient partition that minimizes the total cost of facilities and access over all jurisdictions and is thus Pareto efficient for the entire population. However, since the total cost is infinite, an operational definition invokes cost minimization over bounded sets.

Definition: A partition $P$ of $\Re$ is called efficient if and only if total costs over any finite union of jurisdictions in $P$ are minimal.

A full characterization of efficient partitions is given by:

Lemma 1: A partition $P$ of $\Re$ is efficient if and only if every jurisdiction $S \in P$ is an interval of length $s^{*}$.

In our framework efficiency is implied by secession-proofness, namely, if a solution $(P, x)$ is secession-proof, then the partition $P$ is efficient.

The logic of the proof of the theorem is simple. First, secession-proofness of the Rawlsian allocation $x_{R}^{*}$ associated with the efficient partition $P^{*}$ follows from the fact that no jurisdiction of a non-optimal size could guarantee each of its members a lesser cost burden than

\footnotetext{
${ }^{6} \mathrm{~A}$ partition of the real line into intervals of size $s^{*}$ is only unique up to translations. But if one endpoint of any interval in the partition is fixed, the uniqueness is strict.
} 
that assigned by the optimal jurisdiction. Second, if there exists a secession-proof allocation $x$ which is not Rawlsian, then there exists a set of positive measure, over which individuals contribute finitely less on average under $x$ than under the Rawlsian allocation. The next step is to show the existence of a "small" interval $[a-l, a)$ of "subsidized" individuals, whose endpoint, say, $a$, is also an endpoint of an existing jurisdiction. ${ }^{7}$ The proof then proceeds by contradiction. Consider the interval of optimal size $\left[a-s^{*}, a\right)$. By leaving out a set of "subsidized' individuals $[a-l, a)$ under $x$, the remaining group $\left[a-s^{*}, a-l\right)$ would be better off on its own rather than under the allocation $x$. Indeed, since $s^{*}$ is the optimal jurisdiction size, a tiny shrinkage of the optimal jurisdiction at the boundary has a second-order has a second-order effect in raising the average contribution. However, the savings from leaving out "free riders" are of the first order, and, accordingly, the interval $\left[a-s^{*}, a-l\right)$ is prone to secession when $l$ is sufficiently small. The concluding part of the proof formalizes that intuition by comparing the total costs over a finite but large interval of the size $(N+1) s^{*}-l$ under $x$ and under a partition into $N$ jurisdictions of equal size close (but not equal) to $s^{*}$.

\section{Proof of the theorem}

Part "if". Let us prove that the solution $\left(P^{*}, x_{R}^{*}\right)$ is secession-proof. As shown in the proof of Lemma 1, the total cost for any jurisdiction $S$, if it secedes, is no less than $g+\frac{(\lambda(S))^{2}}{4}$, entailing a cost per citizen no less than $\frac{g}{\lambda(S)}+\frac{\lambda(S)}{4}$. This quantity is minimized at $\lambda(S)=2 \sqrt{g}$, yielding the minimal value of $\sqrt{g}$. However, given $\left(P^{*}, x_{R}^{*}\right)$, the total contribution of members of $S$ is $\lambda(S) \sqrt{g}$, thus leaving no room for beneficial secession by any $S$.

Part "only if". Let us demonstrate that the Rawlsian allocation $x_{R}^{*}$ in the infinite society $\Re$ is the (essentially) unique secession-proof cost allocation. Assume that there exists another secession-proof cost allocation $y$ in $\Re$ that differs from $x_{R}^{*}$ on a set of positive measure. Since the per capita total cost in $\Re$ determined through allocation $x$ is $\sqrt{g}$, there is a group

\footnotetext{
${ }^{7}$ This step adds to the length of the proof. The step, however, would be redundant if a continuity of cost allocations is imposed.
} 
$S$ with $\lambda(S)>0$, such that

$$
y(t)+|t-m(S(t))|<\sqrt{g}
$$

for all $t \in S$. Without loss of generality, we can assume that $S$ lies in the interior of a a jurisdiction $\left(t^{\prime}, t^{\prime}+s^{*}\right)$ in $P$. We then show that there exists an interval $I$ with a positive measure and a positive constant $\delta$ such that the average contribution of members of $I$ assigned by the allocation $y$ is by, at least, $\delta$ below the Rawlsian level, i.e.,

$$
\int_{I}(y(t) d t+|t-m(S(t))|) d t<(\sqrt{g}-\delta) \lambda(I) .
$$

To proceed, we will use the following two lemmas.

Lemma 2: Let $z$ be a secession-proof allocation associated with partition $P, \delta>0$ and $S \subset O$ where $O$ is an open set. Assume that $z(t)<\sqrt{g}-\delta$ a.e. on $S$. Then there exists a finite family of pairwise disjoint intervals $\mathcal{I}=\left\{I_{1}, \ldots, I_{m}\right\}$ with $I_{i} \subseteq O$ for all $I_{i} \in \mathcal{I}$ and $\lambda\left(\bigcup_{I_{i} \in \mathcal{I}} I_{i}\right) \geq \frac{\lambda(S)}{2}$ such that

$$
\int_{I_{i}} z(t) d t<\int_{I_{i}}\left(x_{L}^{R}(t)+|t-m(S(t))|\right) d t-\frac{\delta}{2} \lambda\left(I_{i}\right) \text { for every } I_{i} \in \mathcal{I} \text {. }
$$

Lemma 3 is known as the Lusin theorem (Billingsley (1995), Result 17.10, p. 230):

Lemma 3: Let $A$ be a bounded measurable subset of $\Re$ and $h$ be a measurable function on $A$. Then for every $\varepsilon>0$ there exists a compact set $C_{\varepsilon} \subseteq A$ with $\lambda\left(\tilde{C}_{\varepsilon}\right) \geq \lambda(A)-\varepsilon$ and on which $h$ is continuous.

By Lemma 3, there exists a compact set $C \subseteq S$ with $\lambda(C) \geq \frac{\lambda(S)}{2}$, such that the function

$$
u(t)=y(t)+|t-m(S(t))|
$$

is continuous on $C$. Thus, there exists $\delta>0$ such that $u(t)<\sqrt{g}-\delta$ for all $t \in C$. By applying Lemma 2 to the set $C$, we obtain the existence of an interval $I^{*}=[\bar{t}, \bar{t}+l)$ in the interior of a jurisdiction $J=\left[t^{\prime}, t^{\prime}+s^{*}\right)$ in $P$ with $l>\frac{\lambda(S)}{4 m}$, where $m$ is a finite integer, and

$$
\frac{\int_{I^{*}} u(t) d t}{\lambda\left(I^{*}\right)}<\sqrt{g}-\frac{\delta}{2} .
$$


Then

$$
\frac{\int_{J \backslash I^{*}} u(t) d t}{\lambda\left(J \backslash I^{*}\right)}>\frac{2 g-\lambda\left(I^{*}\right)\left(\sqrt{g}-\frac{\delta}{2}\right)}{s^{*}-\lambda\left(I^{*}\right)}=\sqrt{g}+\gamma, \text { where } \gamma=\frac{\lambda\left(I^{*}\right) \frac{\delta}{2}}{s^{*}-\lambda\left(I^{*}\right)}>0 .
$$

Obviously,

$$
\max \left[\frac{\int_{\bar{t}-t^{\prime}} u(t) d t}{\bar{t}-t^{\prime}}, \frac{\int_{t^{\prime}+s^{*}-\bar{t}-l} u(t) d t}{t^{\prime}+s^{*}-\bar{t}-l}\right]>\sqrt{g}+\gamma .
$$

Assume, without loss of generality, that the maximum above is attained for the interval $\left[t^{\prime}, \bar{t}\right)$. Take a positive integer $N$ and the interval $A=\left[t^{\prime}-N s^{*}, \bar{t}\right)$. Consider a partition $P_{A}$ of $A$ into $N$ jurisdictions of equal size $s^{*}+\frac{\Delta t}{N}$, where $\Delta t \equiv \bar{t}-t^{\prime}$. Then $P_{A}$ generates the total cost $c\left(P_{A}\right)$ over $A$, where

$$
c\left(P_{A}\right)=N\left(g+\frac{1}{4}\left(s^{*}+\frac{\Delta t}{N}\right)^{2}\right)=2 N g+\sqrt{g} \Delta t+\frac{(\Delta t)^{2}}{4 N} .
$$

On the other hand, the total contribution of citizens of $A$ under $y$ satisfies:

$$
\int_{A} u(t) d t>2 N g+(\sqrt{g}+\gamma) \Delta t
$$

It is easy to verify that the last expression exceeds $\left.c\left(P_{A}\right)\right)$ provided $\gamma>\frac{\Delta t}{4 N}$, i.e., $N$ is sufficiently large. This implies that, relatively to the allocation $y$, the interval $A$ can guarantee its citizens a lower total contribution. Thus, there exists a jurisdiction $S$ in $P_{A}$, that can make each of its members better off with respect to $y$. Then $S$ is prone to secession, a contradiction to the assumed secession-proofness of $y$. $\square$

\section{Appendix}

Proof of Lemma 1: Let $P$ be an efficient partition. Then every individual is assigned to her closest facility, which implies that every jurisdiction in $P$ is an interval. Let us demonstrate that all jurisdictions are of the same length. Note that the value of $D(S)$ when $S$ is an interval of size $s$, is given by $\int_{S}|t-m(S)| d t=\frac{s^{2}}{4}$. Let $S$ and $S^{\prime}$ be two adjacent intervals in $P$ and of the length $s$ and $s^{\prime}$, respectively. Since $P$ is efficient, $D(S)+D\left(S^{\prime}\right)$ is minimal among all possible partitions of $S \cup S^{\prime}$ into two intervals, yielding $s^{2}+\left(s^{\prime}\right)^{2} \leq x^{2}+y^{2}$ 
for all nonnegative numbers $x, y$ satisfying $x+y=s+s^{\prime}$. Then the convexity of the function $x^{2}+y^{2}$ immediately implies $s=s^{\prime}$.

To show that the partition $P$ that consists of intervals of the length $s^{*}$ is efficient, it suffices to demonstrate that every partition $P^{\prime}$ into equal intervals of length $s \neq s^{*}$ is inefficient. Consider the case where $s>s^{*}$ (the case $s<s^{*}$ is treated in a similar manner). Since $\frac{s}{s^{*}}>1$, there exists a rational number $\frac{m}{n}$ such that $\frac{s}{s^{*}}>\frac{m}{n}>1$. Thus, there exists $\tilde{s}$ with $s>\tilde{s}>s^{*}$ such that $\tilde{s} m=s n$. Consider partition $\tilde{P}$ obtained by replacing $n$ adjacent intervals of length $s$ in $P^{\prime}$ by $m$ adjacent intervals of length $\tilde{s}$. Put $L=\tilde{s} m=s n$. Since the function $f(t)$ is increasing for $t \geq s^{*}$, and $s>\tilde{s}>s^{*}$, it follows that $L f(\tilde{s})<L f(s)$. However, this inequality is equivalent to $m g+m \frac{\tilde{s}^{2}}{4}<n g+n \frac{s^{2}}{4}$. Since partitions $P^{\prime}$ and $\tilde{P}$ differ only over the finite union of jurisdictions in $P$, the definition of an efficient partition immediately implies than only $P$ is efficient.

Before proceeding with the proof of Lemma 2, we use two claims. The first (from basic measure theory) relies on the regularity of the Lebesgue measure (Billingsley (1995), Theorem 12.3, p.174):

Claim 1: Let $S$ be a bounded measurable subset of $\Re$. Then for every $\varepsilon>0$ there exist a closed set $K_{\varepsilon}$ and an open set $O_{\varepsilon}$ such that $K_{\varepsilon} \subseteq S \subseteq O_{\varepsilon}$ and $\lambda\left(O_{\varepsilon} \backslash K_{\varepsilon}\right)<\varepsilon$.

The next claim states the property of essential boundedness of secession-proof allocations in a multi-jurisdictional framework.

Claim 2: Let $x$ be a secession-proof allocation i associated with a partition $P$ into, at least, two jurisdictions. Then $x(t) \leq 2 s^{*}$ almost everywhere on $\Re$.

Proof: Let $x$ be a secession-proof allocation associated with a partition $P$ that consists of more than one jurisdiction. Denote $S \equiv\left(\left\{t \in \Re: x(t)+|t-m(S(t))|>2 s^{*}\right\}\right)$. We shall show that $\lambda(S)=0$. Suppose, to the contrary, that $\lambda(S)>0$. Let $S_{1}, S_{2}$ be two adjacent jurisdictions in partition $P$ with $\lambda\left(S^{\prime}\right)>0$, where $S^{\prime}=S_{2} \cap S$. Denote $T \equiv S_{1} \cup S^{\prime}$ and 
consider the $T$-cost allocation $y$ that coincides with $x$ on $S_{1}$ and is equal to zero over $S^{\prime}$. We have

$D(T)+g=\int_{T}(y(t)+|t-m(T)|) d t \leq \int_{T}\left(y(t)+\left|t-m\left(S_{1}\right)\right|\right) d t=\int_{S_{1}} x(t) d t+\int_{T} \mid t-m\left(\left(S_{1}\right) \mid d t\right.$.

Since $\left|t-m\left(S_{1}\right)\right|<2 s^{*}<x(t)+\left|t-m\left(S_{2}\right)\right|$ for all $t \in S^{\prime}$, it follows that

$$
\int_{S^{\prime}} \mid t-m\left(\left(S_{1}\right) \mid d t<\int_{S^{\prime}}\left(x(t)+\left|t-m\left(S_{2}\right)\right|\right) d t\right.
$$

Thus,

$D(T)+g<\int_{T} x(t) d t+\int_{S_{1}} \mid t-m\left(\left(S_{1}\right)\left|d t+\int_{S^{\prime}}\right| t-m\left(\left(S_{2}\right) \mid d t=\int_{T}(x(t)+|t-m(S(t))|) d t\right.\right.$.

That is, $T$ is prone to secession, contradicting secession-proofness of $x$.

Proof of Lemma 2: Let $z$ be a secession-proof allocation and let $S \subset O$ be such that $z(t)<\sqrt{g}-\delta$ a.e. on $S$, where $\delta$ is a positive number. Choose $\eta$ such that $0<\eta<\frac{\delta}{8 \sqrt{g}}$. Claim 1 implies that there exists a compact subset $K$ of $S$ such that $\lambda(K)>\frac{3}{4} \lambda(S)$ and an open set $O_{\eta}$ with $O \supseteq O_{\eta} \supseteq S$ such that $\lambda\left(O_{\eta} \backslash K\right) \leq \eta \lambda(K)$.

For every $t \in K$, let $I(t) \subset O_{\eta}$ be an open interval that contains $t$. Since $K$ is compact, the cover $\{I(t)\}_{t \in K}$ admits a finite subcover $\tilde{\mathcal{I}}$. We may assume, without loss of generality, that all intervals in $\tilde{\mathcal{I}}$ are pairwise disjoint, and, moreover, that $O_{\eta}$ consists only of elements of $\tilde{\mathcal{I}}$. Denote by $\mathcal{I}=\left\{I_{1}, \ldots, I_{m}\right\}$ the following subset of $\tilde{\mathcal{I}}$ :

$$
\mathcal{I}=\left\{I_{i} \in \tilde{\mathcal{I}} \mid \frac{\lambda\left(K^{c} \cap I_{i}\right)}{\lambda\left(I_{i}\right)} \leq 5 \eta\right\} .
$$

Thus, we have

$$
\sum_{I_{i} \in \tilde{\mathcal{I}}} \lambda\left(K^{c} \cap I_{i}\right)=\lambda\left(O_{\eta} \backslash K\right) \leq \eta \lambda(K)<\eta \lambda(S) .
$$

But

$$
\sum_{I_{i} \in \tilde{\mathcal{I}}} \lambda\left(K^{c} \cap I_{i}\right)=\sum_{I_{i} \in \mathcal{I}} \lambda\left(K^{c} \cap I_{i}\right)+\sum_{I_{i} \notin \mathcal{I}} \lambda\left(K^{c} \cap I_{i}\right)>5 \eta \sum_{I_{i} \notin \mathcal{I}} \lambda\left(I_{i}\right) .
$$


Thus,

$$
\lambda\left(\bigcup_{I_{i} \notin \mathcal{I}} I_{i}\right)<\frac{\lambda(S)}{5}<\frac{4}{15} \lambda(K), \quad \text { and }\left(\lambda\left(\bigcup_{I_{i} \in \mathcal{I}} I_{i}\right)\right)>\frac{11}{15} \lambda(K)>\frac{\lambda(S)}{2} .
$$

Let $I=\bigcup_{I_{i} \in \mathcal{I}}$. We have

$$
\int_{I} z(t) d t=\int_{K \cap I} z(t) d t+\int_{K^{c} \cap I} z(t) d t, \text { where } \int_{K \cap I} z(t) d t<\lambda(I)(\sqrt{g}-\delta) .
$$

By Claim $2, z(t)<2 s^{*}=4 \sqrt{g}$ almost everywhere on $\Re$, yielding $\int_{K^{c} \cap I} z(t) d t \leq 4 \sqrt{g} \eta \lambda(I)$. But $\eta<\frac{\delta}{8 \sqrt{g}}$ implies $\int_{I} z(t) d t<\lambda(I)\left(\sqrt{g}-\frac{\delta}{2}\right)$, which completes the proof of the lemma.

\section{References}

Alesina, A. and E. Spolaore (1997) "On the Number and Size of Nations", Quarterly Journal of Economics 113, 1027-1056.

Aumann, R. and J. Drèze (1974) "Cooperative Games with Coalition Structure", International Journal of Game Theory 3, 217-237.

Billingsley, P. (1995) Probability and Measure, third edition. Wiley, New York.

Haimanko, O., Le Breton, M. and S. Weber (2004) "Voluntary Formation of Communities for the Provision of Public Projects", Journal of Economic Theory 115, 1-34.

Le Breton, M. and S. Weber (2003) "The Art of Making Everybody Happy: How to Prevent a Secession?", IMF Staff Papers 50 (3), 403-435.

Le Breton, M., Weber, S. and J. Drèze (2004) "The Rawlsian Principle and SecessionProofness in Large Heterogeneous Societies", CORE Discussion Paper 2004/61. 